\title{
Design and Evaluation of a Novel Trackball Input Device for Middle-Aged Users
}

\author{
Fong-Gong Wu, Jack Chuang, Chien-Hsu Chen, and Li-Ru Lai \\ Department of Industrial Design, National Cheng Kung University, Tainan, Taiwan \\ \{fonggong, chenhsu\} @mail.ncku. edu.tw, \\ \{jack.chuang, lai1203\}@gmail.com
}

\begin{abstract}
This study developed two sets of novel trackball input devices operated by two hands with no space constraints, and required a series of experiments. Besides, the performances of the two newly-developed devices are significantly better than both existing trackball input devices in several tasks. However, the results of stability assessment tests show that the ability of a mouse to control the cursor is still greater than all the other devices. In physical observation part, wrist extension and ulnar deviation were measured while using Jack-1 and Jack-2, and showed apparently slighter than all the other devices.
\end{abstract}

Keywords: Trackball input device, Two-handed operation, Middle-aged people.

\section{Introduction}

In the recent years, the development of Graphical User Interface, GUI such as intuitive-operating icon or windows has led to effortless-operation without the use of keyboards. This has increased the operation time of Non-Keyboard Input Devices, NKID. Amongst which, computer mouse is still the most popular device employed by the current computer users [1][2][3][4]. Experiment results from past studies have proved that computer mouse is easier to operate than most other non-keyboard input devices with higher efficiency (including accuracy and speed). However, mouse operation employs the elbow as the fulcrum, integrating the motion of forearm, upperarm, and fingers, resulting in shoulder abduction, flexion, dorsiflexion, ulnar deviation, radial deviation, and musculoskeletal disorders, the so called Cumulative Trauma Disorders, CTDs [5][6]. Therefore, many researchers make attempts to improve the current input devices or replacement input devices for different user groups to avoid this problem, hoping for a suitable input device in the future that is both comfortable and efficient [7][8].

Aside from computer mouse, the trackball input device is another commonly employed control input device [9]. Because of this, it is often compared to the computer mouse for control efficiency performance and comfort levels. Results of related studies in the past pointed out that operating computer mouse and trackball mouse both lead to dorsiflexion and ulnar deviation. However, in comparison to the computer mouse, the use of the trackball mouse results in less cases of wrist dorsiflextion and shoulder abduction occurrence. As for the comparison between 
different age groups, according to the study of [10][11], user groups of older ages prefer the trackball mouse for its smaller wrist movement range even though it is less efficient than the computer mouse. It is an operational input device suitable for the elders.

The research purpose is to confer and integrate suggestions to improve the trackball input device from past research results in order to investigate possible developments of the trackball input device. The researcher hopes to retain strengths in the current design and improve on the weakness in operation manners to improve on efficiency, hence develop a trackball input device with efficiency and comfort which conforms to human factors. Furthermore, the study specifies the middle-aged user group, run experiments with different operations to assess and observe body motions and compare operation efficiency with the current computer mouse and trackball input devices. In this study, the trackball input device is chosen to be the goal of development and improvement specified for the middle-aged user group of users between 45 and 60 years of age. Furthermore, this study designs operation experiments regarding the common operations such as pointing, clicking, and dragging, in order to assess the efficiency of different input devices and observe body motions and posture of the participants by using the Motion-Capture system.

\section{Methods}

\subsection{Design Development}

Through the observation of interview participants (5 participants) operating six current trackball input devices, strengths and weaknesses of each device were found and employed as related design parameters. The results of observations and interviews show that Track Mouse and The Fish, with similar trackball sizes, present the best trackball size. One participant claimed that Iball was the best but would be better if it was flatter. Another participant thought that the best trackball size should be between the size of trackball on the TrackMan and The Fish. All five participants thought the trackball on Pen Mouse was too small. As for the sensitivity of trackballs, all participants thought that Marble Mouse, TrackMan and Iball were the best ones while Pen Mouse was thought to be the least sensitive. Regarding the shape of devices, four participants thought Track Mouse was difficult to hold; three participants thought The Fish was comfortable to hold; and one thought Iball was too big to hold therefore employed both hands. Also, all five participants expressed their fondness for The Fish, Pen Mouse, and Track Mouse for their freedom of space confinement. As a whole, Marble Mouse and The Fish were thought to be the best devices. Integrating past literature and the results of practical observation and interviews, the ideal design objectives and criteria have been planned and shown in Fig. 1. Following the above design objectives and criteria, different operation posture (Fig. 2) and operation manners are integrated to develop a number of different design concepts (shown in fig. 3). Finally, through discussions with professionals, tests, and selections, two final design prototypes were produced (shown in Fig. 3 as Jack-1 and Jack-2). 


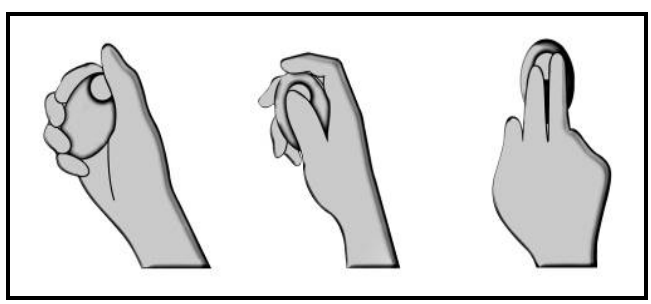

Fig. 1. Different hand posture concepts

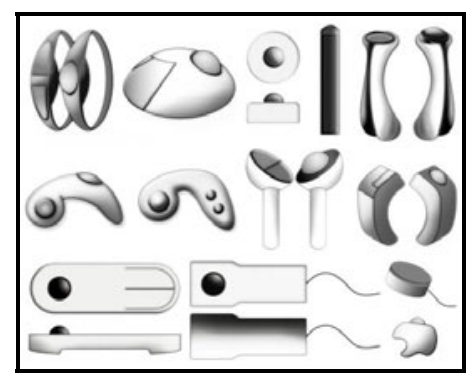

Fig. 2. Design concept development

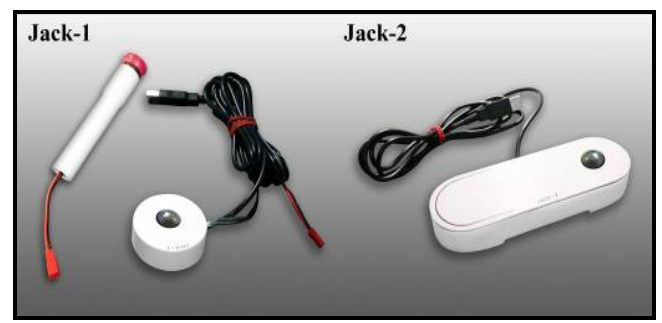

Fig. 3. Final design prototypes

\subsection{Experiment Design}

Ten healthy participants between ages 45 60 with no muscle or bone injuries were selected to investigate the operation efficiency of middle-aged users operating input devices. Participants were put through different combinations of tests to evaluate the operation efficiency of each device. Each participant were given 5 10 minutes of practice time to get acquainted with the input devices and adjust the working environment according to their own preferences, such as table height, distance between body and table, chair back angle, screen distance, screen angle, and screen brightness etc.

This research is aimed at comparing the operation efficiency of the five input devices when facing different operations. The devices are: a. Optical Mouse by S+ARCK, b. Logitech Marble Mouse, c. The Fish hand-held Trackball Mouse, d. Jack-1, e. Jack-2.

The efficiency evaluation of three combinations of operations is included in this research, such as: Point and Select task assessment test, Dragging task assessment test, and Stability task assessment experiment.

- Point and Select Task Assessment Experiment. This experiment requires participants to move the cursor to the target area for clicking tasks. First, the cursor needs to be at the original point area and the "start" button needs to be pressed to begin the task. At this point, target area 1 would appear on the screen and participants are required to move the cursor to click number 1 . Next, target area 0 would appear on the screen and participants are required to move the cursor to 
click number 0 . This movement is repeated three times and a total of 8 target areas located in different directions are required to be clicked. The distance (D) between the original point to the target area is stable, and the shifting of angles follows a clockwise direction. This point and select task is operated with five different input devices and three different target area sizes. The experiment chart is showing in fig. 4.

- Dragging Task Assessment Experiment. This experiment requires participants to drag target objects in the target areas to the next using the cursor. First, move the cursor to the original point and click on the "Start" button to begin. At this point, participants are required to click and hold the key to move the target object in the original point area (number 0 target area) and drag the object to the number 1 target area, then drop the object by releasing the key. Next, the number 0 target area would reappear on the screen and participants are required to repeat the same dragging movement to bring the target object back to the 0 target area. This movement is repeated three times with 8 target areas in different directions. This task is operated with five different input devices and three different target area sizes. The experiment chart is showing in fig. 5.

- Stability Task Assessment Experiment. This experiment requires participants to drag the cursor from "Start" and follow the given paths clockwise. This task contains three different paths each bordered with two sidelines $10 \mathrm{~mm}$ apart. Tracks are left after the dragging of cursors for further analysis. In cases where cursors touch the sidelines, the computer gives a warning sound and keeps a record. Since the point of the task is to assess the stability of the input devices, the participants are informed to avoid touching the sidelines. What this means, is that stability is the top priority rather than speed. The experiment chart is showing in fig. 6 .

\section{Analysis and Results}

After the experiment of evaluating the tests (Point and select, Dragging operation and stability) and observing the body movements, all of the data are collected and collated for analysis and discussion. This study includes a total of ten participants. The effective sample is 10 . Furthermore, 5 subjects from these 10 are selected to be tested by using interception system test for body observation.

\subsection{Point and Select Task Assessment Analysis}

After all the data were collected, the researcher ran the One-Way ANOVA by SPSS 13.0 to analyze the data. It is revealed that the average time required was significant $(p<0.05)$ when the researcher manipulated different kinds of NKID to process all the achievement evaluation tests. However, with error frequency, it is only significant $(\mathrm{p}<0.05)$ with different NKID.

In point-and-select test 1 ,it was suggested that Mouse required the shortest time, which is significantly different from other devices, while The Fish required the longest time, demonstrating a significant difference, as shown in the Table 1 . In the aspect of errors, it was absent when with all participants using Jack-2. The researcher analyzed the distribution of errors at different angles with different NIKD. 


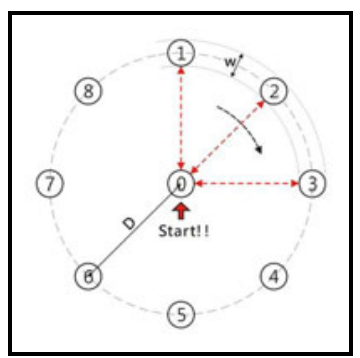

Fig. 4. Point and select task test chart

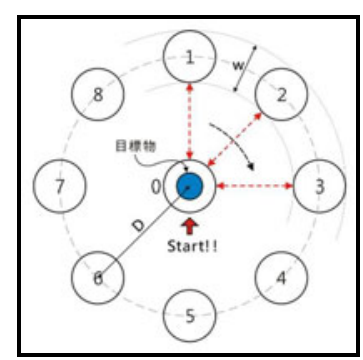

Fig. 5. Dragging task experiment chart

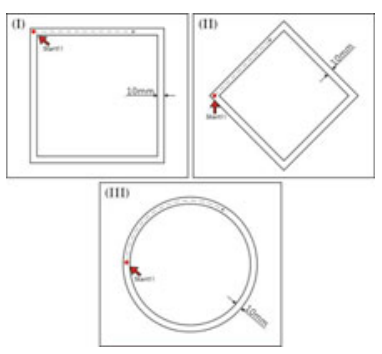

Fig. 6. Stability task assessment chart

In point-and-select test 2 , it is suggested that Mouse required the shortest time, which is significantly different from other devices in terms of testing time, while The Fish required the longest time, demonstrating a significant difference, as shown in the Table 2. Mouse and Jack-2 had the least errors amongst all devices.

In point-and-select test 3 , it is suggested that Mouse required the shortest time, which is significantly different from other devices in terms of testing time, while The Fish requires the longest time, demonstrating a significant difference, as shown in the Table 3. Jack-2 had the least errors amongst all devices. The researcher analyzed the distribution of errors when every NIKD processed point-and-select test 3 by each angle.

Table 1. Post Hoc - Point and select test 1 experiment time

\begin{tabular}{lllll}
\hline Duncan & \multicolumn{3}{l}{ Subset for alpha $=.05$} \\
\hline Input Device & Test sample $(\mathrm{N})$ & 1 & 2 & 3 \\
\hline Mouse & 10 & 35.40 & & \\
Jack-1 & 10 & & 52.70 & \\
Jack-2 & 10 & & 54.10 & \\
Marble & 10 & & 59.30 & \\
The Fish & 10 & & & 76.70 \\
\hline Sig. & & 1.000 & .195 & 1.000 \\
\hline
\end{tabular}

Table 2. Post Hoc - Point and select test 2 experiment time

\begin{tabular}{|c|c|c|c|c|}
\hline \multicolumn{2}{|l|}{ Duncan } & \multicolumn{3}{|c|}{ Subset for alpha $=.05$} \\
\hline Input device & Test samples(N) & 1 & 2 & 3 \\
\hline Mouse & 10 & 40.10 & & \\
\hline Jack-1 & 10 & & 59.20 & \\
\hline Jack-2 & 10 & & 60.00 & \\
\hline Marble & 10 & & 64.80 & \\
\hline The Fish & 10 & & & 85.50 \\
\hline Sig. & & 1.000 & .322 & 1.000 \\
\hline
\end{tabular}


Table 3. Post Hoc - Point and select test 3 experiment time

\begin{tabular}{lclcl}
\hline Duncan & & \multicolumn{3}{l}{ Subset for alpha $=.05$} \\
\hline Input device & Test sample $(\mathrm{N})$ & 1 & 2 & 3 \\
\hline Mouse & 10 & 45.60 & & \\
Jack-2 & 10 & & 69.00 & \\
Jack-1 & 10 & & 70.10 & \\
Marble & 10 & & 83.80 & \\
The Fish & 10 & & & 101.80 \\
\hline Sig. & & 1.000 & .056 & 1.000 \\
\hline
\end{tabular}

\subsection{Dragging Evaluation Test}

In dragging test 1 it is suggested that Mouse required the shortest time, which is significantly different from Marble and The Fish, but not significant in Jack-1 and Jack-2, in terms of testing time. The Fish required the longest time, demonstrating a significant difference from Jack-1 and Jack-2, as shown in the Table 4. Mouse and Jack-2 had the least errors in all devices. The researcher analyzed the distribution of errors from different angles.

Table 4. Post Hoc - Dragging test 1 experiment time

\begin{tabular}{lllll}
\hline Duncan & \multicolumn{4}{l}{ Subset for alpha $=.05$} \\
\hline Input device & Test samples (N) & 1 & 2 & 3 \\
\hline Mouse & 10 & 63.90 & & \\
Jack-2 & 10 & 88.4000 & & \\
Jack-1 & 10 & 96.6000 & 96.60 & \\
Marble & 10 & & 123.90 & 123.90 \\
The Fish & 10 & & & 144.20 \\
\hline Sig. & & .065 & .103 & .223 \\
\hline
\end{tabular}

In dragging test 2 , it is suggested that Mouse required the shortest time, which is significantly different from Jack-1 and Jack-2, but these three showed a significant difference in The Fish and Marble. Marble required the longest time in all devices, not significant from The Fish, as shown in the Table 5. In terms of errors, Jack-1 and Jack-2 had the least errors amongst all. The researcher analyzed the distribution of errors from each angle.

In dragging test 3 , it is suggested that Mouse required the shortest time, which is significantly different from Jack-1 and Jack-2, but these three showed a significant difference in The Fish and Marble. The Fish required the longest time amongst all devices, not significant from Marble, as shown in Table 6. Jack-1 had the least error frequency amongst all devices. The researcher analyzed the distribution of errors when every NIKD processed dragging test 3 from different angles. It is revealed that the Marble and The Fish had the most errors of 75 and 112 respectively. 
Table 5. Post Hoc - Dragging test 2 experiment time

\begin{tabular}{lllc}
\hline Duncan & & \multicolumn{2}{l}{ Subset for alpha $=.05$} \\
\hline Input device & Test samples $(\mathrm{N})$ & 1 & 2 \\
\hline Mouse & 10 & 62.90 & \\
Jack-1 & 10 & 88.30 & \\
Jack-2 & 10 & 89.30 & 148.30 \\
The Fish & 10 & & 149.30 \\
Marble & 10 & & .948 \\
\hline Sig. & & .106 & \\
\hline
\end{tabular}

Table 6. Post Hoc - Dragging test 3 experiment time

\begin{tabular}{llll}
\hline Duncan & & \multicolumn{2}{l}{ Subset for alpha $=.05$} \\
\hline Input device & Test samples $(\mathrm{N})$ & 1 & 2 \\
\hline Mouse & 10 & 65.90 & \\
Jack-1 & 10 & 98.00 & \\
Jack-2 & 10 & 100.60 & 157.00 \\
Marble & 10 & & 193.50 \\
The Fish & 10 & & .128 \\
\hline Sig. & & .171 & \\
\hline
\end{tabular}

\subsection{Stability Task Assessment Analysis}

In stability test 1 , it is suggested that Mouse required the shortest time, which is significantly different from other devices, while Jack-2 required the longest time, showing an insignificant difference in Marble, Jack-1 and The Fish, as shown in the Table 7.

In stability test 2 , it is suggested that Mouse required the shortest time, which is significantly different from other devices, while The Fish required the longest time, showing an insignificant difference in Jack-1 and Jack-2, as shown in the Table 8. In terms of errors, The Fish had the most errors, but Mouse had the least errors, which is not significant from Jack-1, Jack-2 and Marble.

In stability test 3 , it is suggested that Mouse required the shortest time, which is significantly different from other devices, while The Fish required the longest time, showing an insignificant difference in Jack-1 and Jack-2, as shown in the Table 9. In terms of errors, The Fish had the most errors, but Mouse had the least errors, which is only significantly different from The Fish, but insignificant different from Jack-1, Jack-2 and Marble. 
Table 7. Post Hoc - Stability test 1 experiment time

\begin{tabular}{llll}
\hline Duncan & & \multicolumn{2}{l}{ Subset for alpha $=.05$} \\
\hline Input device & Test samples $(\mathrm{N})$ & 1 & 2 \\
\hline Mouse & 10 & 14.00 & \\
Marble & 10 & & 21.20 \\
Jack-1 & 10 & & 21.80 \\
The Fish & 10 & & 22.10 \\
Jack-2 & 10 & 1.000 & 22.30 \\
\hline Sig. & & & .733 \\
\hline
\end{tabular}

Table 8. Post Hoc - Stability test 2 experiment time

\begin{tabular}{|c|c|c|c|c|}
\hline \multicolumn{2}{|l|}{ Duncan } & \multicolumn{3}{|c|}{ Subset for alpha $=.05$} \\
\hline Input device & Test samples (N) & 1 & 2 & 3 \\
\hline Mouse & 10 & 17.80 & & \\
\hline Marble & 10 & & 27.30 & \\
\hline Jack-2 & 10 & & 30.90 & 30.90 \\
\hline Jack-1 & 10 & & 31.00 & 31.00 \\
\hline The Fish & 10 & & & 37.50 \\
\hline Sig. & & 1.000 & .383 & .121 \\
\hline
\end{tabular}

Table 9. Post Hoc - Stability test 3 experiment time

\begin{tabular}{lllll}
\hline Duncan & \multicolumn{3}{l}{ Subset for alpha $=.05$} \\
\hline Input device & Test samples $(\mathrm{N})$ & 1 & 2 & 3 \\
\hline Mouse & 10 & 12.50 & & \\
Marble & 10 & & 21.50 & \\
Jack-1 & 10 & & 23.90 & 23.90 \\
Jack-2 & 10 & & & 27.60 \\
The Fish & 10 & & & 28.80 \\
\hline Sig. & & 1.000 & .390 & .100 \\
\hline
\end{tabular}

The test performance assessment results of the research show that Mouse showed outstanding performance on the test of average time. This is particularly evident in the point and select and the stability tests. From the point and select task assessments we can see that the average time for task completion using Mouse is about 20 seconds shorter than the device with the second shortest completion time (Jack-1 or Jack-2). However, the speed of task operation resulted in the lack of outstanding performance in the error rate assessment. In the point and select test 1, the newly developed devices Jack-1 and Jack-2, even Marble with the trackball input device, all performed better than the Mouse in the error rate test. Also, in the point and select test 3, Jack-2 with the lowest error rate amongst all devices also performed better in the average error rate compared to the Mouse. Other than that, there was no significant difference between the error rate in the point and select test for all devices at different angles. 
In the dragging task assessment, there was no significant difference between Jack1, Jack-2 and the Mouse. In dragging test 2 and 3, the average time for Jack-1 and Jack-2 were both significantly shorter than the current trackball input devices Marble and The Fish. In dragging test 1, the average time performance for Jack-2 was significantly shorter than Marble and The Fish. These results show that the appropriate use of both hands does improve the performance of trackball input devices while performing dragging tasks. Also, in the dragging test average error rate, Jack-1 and Jack-2 showed the best and the second best performance. As for the error rate at different angles, all devices resulted in a higher error rate with oblique angles $\left(45^{\circ}, 135^{\circ}, 225^{\circ}\right.$, and $\left.315^{\circ}\right)$. In dragging tests 1 and 3 , the total error frequency for the Fish at $135^{\circ}$ were as high as 25 times.

In the stability assessment tests, since the goal is to assess the stability of devices, the participants were told to put stability as the first priority over speed. Therefore, the average error rate is the item assessed here. From the results of the three stability tests, we can see that the average error rate and average time for the Mouse were both the best. It is clear that Mouse has the best performance in the assessment tests while the other devices show no significant differences amongst them. These results show that even with the improvement of the $\mathrm{C} / \mathrm{R}$ ratio to increase stability, there was not significant improvement in the stability of trackball control. This means even with the control of trackball over the cursor, there is still a significant difference compared to the stability of using Mouse.

\section{Conclusions}

This study is designed for middle-aged users and develops new trackball input devices. Therefore, the operation manners and the level of overall comfort are important elements for improvement. By the appropriate use of both hands to adjust the trackball operation and configuration (including the selection of the appropriate size of the trackball, location and height of the spheroid), along with the operation without space confinement, the appropriate size and feedback button, this study allows users to control in a more relaxed posture with the two newly developed devices. The result shows that when the participants use input devices, their wrists conform to different levels of ulnar deviation, distortion, and dorsiflexion. Unlike Mouse and Marble which are limited by the desk top space and the operation posture, Jack-1 and Jack-2 decrease the level of ulnar deviation and dorsiflexion on participants by about half. For the angle of the elbow, when using the Mouse and Marble, due to the limitation of the desk space and posture, the participants were exposed to an unnatural hand position for a long time which easily causes skeletal muscle injury. Because The Fish and these two newly developed devices are not free of space confinement, participants have the freedom to change their posture to reduce fatigue. In addition, as the operation of Mouse requires the use of the entire arm to control the cursor, participants' elbow angle changes constantly. The other four devices are rendered in a steady angle, which reduces the load on elbow joints.

It is found that improving the level of comfort and feasibility of trackball input devices can increase work efficiency and overall performance. In the experiment, Jack-1 and Jack-2 both performed equally well, however, according to the data from physical observation experiments, when the subjects use Jack-1, the dorsiflexion of 
the non-preferred wrist is more serious than using Jack-2. Also, in the stability tests, it can be found that the participants are not used to the control of cursor with the trackball. From the results of stability test1, the average number of errors occurred for Jack-2 is even higher than The Fish. It shows that there is no significant improvement in the present trackball input devices. It will be the next part for improvement. Additionally, it is believed that designing and coping with the relationship between the form of non-preferred hand and Keyboard to enhance the overall performance of using computer will also be the direction for future development. Finally, the conclusion of this study and design recommendations are expected to provide the direction for future designers who may develop better alternative input devices to provide middle-aged computer users with more options.

\section{References}

1. Jensen, C., Borg, V., Finsen, L., Hansen, K., Juul-Kristensen, B., Christensen, H.: Job demands, muscle activity abd musculoskeletal symptoms in relation to work with the computer mouse. Scandinavian Journal of Work and Environmental Health 24, 418-424 (1998)

2. Woods, V., Hastings, S., Buckle, P., Haslam, R.: Ergonomics of using a mouse or other non-keyboard input device. Health and Safety Executive, Research Report 045 (2002)

3. Atkinson, S., Woods, V., Haslam, R.A., Buckle, P.: Using non-keyboard input devices: interviews with users in the workplace. International Journal of Industrial Ergonomics 33, 571-579 (2004)

4. Donker, A., Reitsma, P.: Drag-and-drop errors in young children's use of the mouse. Interacting with Computers 19, 257-266 (2007)

5. Fogleman, M., Brogmus, G.: Computer mouse use and cumulative trauma disorders of the upper extremities. Ergonomics 38(12), 2465-2475 (1995)

6. Harvery, R., Peper, E.: Surface electromyography and mouse use position. Ergonomics 4(8), 781-789 (1997)

7. Springer, J., Siebes, C.: Position controlled input device for handicapped: experimental studies with footmouse. International Journal of Industrial Ergonomics 17, 135-152 (1996)

8. Betke, M., Gips, J., Fleming, P.: The camera mouse: visual tracking of body features to provide computer access for people with severe disabilities. IEEE Trans. Neural Systems Rehab. Eng. 10, 1-10 (2002)

9. Karlqvist, L., Bernmark, E., Ekenvall, L., Hagberg, M., Isaksson, A., Rostö, T.: Computer mouse and track-ball operation: Similarities and differences in posture, muscular load and perceived exertion. International Journal of Industrial Ergonomics 23, 157-169 (1999)

10. Chaparro, A., Bohan, M., Fernandez, J., Kattel, B., Choi, S.D.: Is the Trackball a Better Input Device for the Older Computer User? Journal of Occupational Rehabilitation 9(1), 33-43 (1999)

11. Chaparro, A., Bohan, M., Fernandez, J., Kattel, B., Choi, S.D.: The impact of age on computer input device use: Psychophysical and physiological measures. International Journal of Industrial Ergonomics 24, 503-513 (1999) 